\title{
Comparative Study on the Rice Bran Stabilization Processes: A Review
}

ISSN: 2576-8840

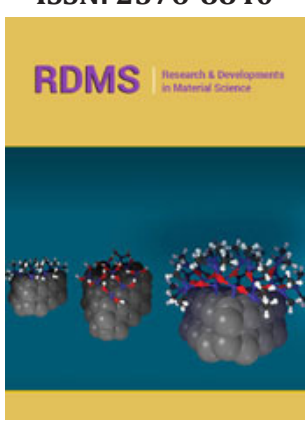

*Corresponding author: : Bipro N Dubey, National Centre of Excellence for Food Engineering, Faculty of Science Technology and Arts, United Kingdom

Submission: 侮July 10, 2019

Published: 監July 15, 2019

Volume 11 - Issue 2

How to cite this article: Bipro N D, Drew F Samsun N, Martin H. A Comparative Study on the Rice Bran Stabilization Processes: A Review. Res Dev Material Sci. 11(2) RDMS.000759.2019.

DOI: 10.31031/RDMS.2019.11.000759

Copyright@ Bipro N Dubey, This article is distributed under the terms of the Creative Commons Attribution 4.0 International License, which permits unrestricted use and redistribution provided that the original author and source are credited.

\section{Bipro N Dubey*, Drew Fitton, Samsun Nahar and Martin Howarth}

National Centre of Excellence for Food Engineering, Faculty of Science Technology and Arts, Sheffield Hallam University, Howard Street, Sheffield S1 1WB, United Kingdom

\begin{abstract}
Rice bran is an undervalued/underutilized by-product of rice milling, rich in protein, lipids, dietary fibers, vitamins, and minerals. It is an inexpensive source of high-quality protein, fiber and lipids to be incorporated into value-added food products. The issue with rice bran is its susceptibility to rancidity and therefore measures must be taken to stabilize the bran in order for it to be fully utilized. Due to this susceptibility to rancidity, historically the bran has either been disposed and wasted or used as low-grade animal feed. As the nutritional value of the bran has been recognized, along with its potential to add value to food products, research has been increasing in volume in order to determine the most effective methods for stabilizing the bran and extracting the valuable nutrients from it. It's been reported that a free fatty acid content of over $5 \%$ is considered to render the bran unfit for human consumption (Tao, Rao \& Liuzzo, 1993). Therefore, controlling this level of rancidity is imperative to being able to store and use rice bran over extended periods of time. In order to achieve control, stabilization procedures can be carried out on the rice bran to slow down the lipase activity within the bran and preserve the nutritional qualities that rice bran possesses. Stabilization of rice bran is particularly important for use over winter months in developing countries, where there may be no crops to harvest and therefore a supply of non- rancid rice bran could be extremely beneficial. This length of storage for stabilized rice bran could be up to a period of 6 months, where it can become important for value-added product development (Bagchi, Adak \& Chattopadhyay, 2014). The present review will provide an overview of the traditional and innovation rice bran stabilization techniques, those have been a common interest in the research community, and the suitability of the process in terms of the energy consumption.
\end{abstract}

Keywords: Rice bran; Rancidity; Stabilization; Microwave heating; Infrared heating; Energy consumption

\section{Introduction}

Rice is a major food product across the globe, it is grown in over 100 countries and more than 1 billion people rely on it in order to survive. Rice constitutes $30 \%$ of the population's diet in developing countries where the average human consumption is around $12 \mathrm{~kg}$ a year. The common form in which rice is consumed is as white rice. The process to produce white rice is known as the rice milling process. In the rice milling process the hard shell known as the husk is first removed, this holds little to no nutritional value. Left is the rice grain and a brown covering, more commonly known as rice bran. The rice bran contains around $65 \%$ of the nutrients within the rice crop (Begum et al., 2015).

Begum et al. (2015) state that around 30 million metric tonnes of rice bran are produced annually as a by-product of the rice milling process, which is either disposed of as waste or used as low-grade animal feed. However, literature shows that rice bran has more opportunities to be used in food production, due to its high nutritional value. The rice bran itself contains 12 $15 \%$ protein and an oil percentage of $14-20 \%$. The oil, which can be extracted from the rice bran is said to have a balanced fatty acid composition, one which renders the oil nutritionally high-quality (Ahmed, Platel, Vishwanatha, Puttaraj \& Srinivasan, 2006). The desirable nature of its properties should, therefore, be utilized, and rice bran should become a major part of high-quality, nutritious food production.

The bran contains many desirable qualities for human consumption such as iron, B vitamins, fibers, lipids, and proteins. Alongside small molecules which are said to be beneficial for preventing, controlling and treating a number of diseases (Borresen \& Ryan, 2014). The health beneficial effects of these small molecules cover antioxidants, anti-inflammatories, 
chemo-preventatives, and gastrointestinal microbe interactions, alongside an abundance of others (Begum et al., 2015). As a result of this information, many studies are being undertaken regarding the effect of rice bran consumption on chronic disease prevention. Some studies concluding that rice bran consumption has the capacity to control cardiovascular disease (Cicero \& Derosa, 2005), lowering cholesterol (Kahlon, Chow, Chiu, Hudson \& Sayre, 1996) and the potential to have a significant impact on cancer prevention for the global population (Henderson et al., 2012).

A large area of interest in the food industry over the past few years has become plant-based foods. A report by Food Network Revolution (2018) claims that there was a 600 percent increase in identifying Vegans in the USA between 2014 and 2017. Baum and Whiteman (2018) claim that "35 percent of Americans get most of their protein from sources other than red meat, and 83 percent of Americans are adding plant-based foods to their diet to improve health and nutrition". With this growing trend comes the demand for plant-based protein as a meat substitute, and one which manufacturers of products utilizing rice bran oil can take advantage of.

A major drawback to rice bran is its susceptibility to becoming rancid in a matter of hours to days. This rancidity is caused by free fatty acid production within the bran, "lipases break the links between fatty acids and glycerol ester, increasing the rate of free fatty acid production" (Gopinger et al., 2015). The free fatty acid content in the rice bran increases within hours and can reach 5-7\% within 24 hours of milling (Ramezanzadeh, 1998). A free fatty acid content of over $5 \%$ is considered to render the bran unfit for human consumption (Tao, Rao \& Liuzzo, 1993). Therefore, controlling this level of rancidity is imperative to being able to store and use rice bran over extended periods of time. In order to achieve control, stabilization procedures can be carried out on the rice bran to slow down the lipase activity within the bran and preserve the nutritional qualities that rice bran possesses. Stabilization of rice bran is particularly important for use over winter months in developing countries, where there may be no crops to harvest and therefore a supply of non-rancid rice bran could be extremely beneficial. This length of storage for stabilized rice bran could be up to a period of 6 months, where it can become important for value-added product development (Bagchi, Adak \& Chattopadhyay, 2014).

The quality of the stabilization process itself must also be considered, with a poorly designed stabilization process, the rice bran may not become rancid, however, the nutritional qualities of the rice bran could be compromised (Irakli, Kleisiaris, Mygdalia \& Katsantonis, 2018). The ideal stabilization process is one which reduces the lipase activity within the rice bran to the lowest possible level, whilst still holding the nutritional value over time. It is also possible to increase some nutritional properties of the rice bran through the use of a stabilization process. Lv, He \& Sun (2018) found that after dry heating and microwave stabilization treatments, the protein purity of the samples increased. With the highest purity yield of $73.34 \%$, an increase of $11.08 \%$ from the control sample.

\section{Stabilization techniques}

In order to understand the effect of stabilization on the properties of rice bran, an analysis of the literature data have been carried out on various stabilization techniques. However, all relevant data found from research into rice bran stabilization based on microwave heating and infrared heating have been extracted and manipulated, and comparative analysis is presented below.

Ohmic heating: Research into ohmic heating hasn't been as prominent as other methods, therefore only a small selection of data is available for analysis and is measured over a 3- to 6-week storage period. However, most of the data fall within the $5 \%$ FFA content which is deemed to be fit for human consumption. Concluded from Lakkakula, Lima \& Walker (2014) and Loypimai, Moonggarm, and Chottanom (2009), is that moisture content of rice bran for ohmic heating is a major factor in the effectiveness of the stabilization treatment. The samples for which the stabilization treatment was deemed ineffective for a 4-week storage period had a native moisture content of $10.5 \%$, whereas when the moisture content was adjusted to $21 \%$ the FFA content of the bran was $4.15 \%$ and $4.40 \%$ after 4 weeks. From the data found in literature it can be concluded that ohmic heating is a viable method for the stabilization of rice bran, however, due to the lack of data available, more research would be advantageous, specifically for a storage period of up to 10 weeks.

Acid treatment: As with ohmic heating, research surrounding acid stabilization for rice bran is not large in volume, and therefore multiple studies for one specific chemical treatment have not been published. The data published by Akhter (2015), where 4 acids at 3 different concentrations were used in the stabilization. Concluding from the data, phosphoric acid has little to no effect on the FFA content in bran at any concentration. Sodium metabisulphite and acetic acid treatments decelerated the FFA increase in the bran, however, all samples exceeded 5\% FFA content within a 2-week storage period. Hydrochloric acid at the highest concentration of $35 \mathrm{ml} / \mathrm{kg}$ was the only treatment which kept the FFA content in the sample below $5 \%$ within 2 weeks of storage. No samples had an FFA content of below $5 \%$ after a 4 -week storage period and therefore would be considered unfit for human consumption.

Dry heating: The use of dry heating methods for stabilization studies found that the FFA content retained to within $10 \%$ in an 8 -week storage period, with $60 \%$ of these under the critical FFA content of $5 \%$ in a 4 -week storage period. When considering the optimum conditions for dry heating, the literature available is not in complete agreeance; Kim (2014) and Amarasinghe (2009) both heated the bran at $100{ }^{\circ} \mathrm{C}$ for 1 hour, however after 4 weeks Amarasinghe (2009) measured a 134\% increase from the FFA content at week 0, whereas Kim (2014) only recorded a $40 \%$ increase over the same time period. To conclude, from the data available in the literature, dry heating is an effective method for rice bran stabilization, disregarding the results from Tao (1989), as they do not align with any other data. Due to the amount of data available being relatively small, more research would be advised in order to 
find the most efficient conditions for the stabilization treatment in order to ensure the FFA content within the bran remains below $5 \%$ after a 4-week storage period.

Microwave heating: Figure 1 below, shows the data (available in the literature) for microwave stabilized rice bran and its free fatty acid content over a storage period of up to 16 weeks. As rice bran with a free fatty acid content exceeding $5 \%$ is deemed to be unfit for human consumption, there are a number of treatments which fail to stabilize the bran to an acceptable level. In order to assess the quality of the treatment method, analysis of the free fatty acid after 4 weeks can be carried out. There are 4 treatments which are anomalies within the data set (Patil et al., 2016), treatments a-c were performed at a microwave power density of $2 \mathrm{~W} / \mathrm{g}$ for 2 , 4 and 6 minutes, the intensity of $2 \mathrm{~W} / \mathrm{g}$ is therefore concluded to be insufficient for stabilizing rice bran. Treatment $d$ was performed at a power density of $4 \mathrm{~W} / \mathrm{g}$ for 2 minutes and therefore concluded that the treatment time was insufficient to stabilize the bran. Disregarding patil's data a-c, where the treatment was considered to be extremely insufficient, when the initial FFA measurement was under $5 \%, 87.5 \%$ of treatments were sufficient to keep the FFA under the acceptable $5 \%$ value after 4 weeks. From the data collected, the results show that dependent on the exact parameters for microwave treatment, it has the potential to provide enough stability in rice bran for up to a 4-week storage period after milling.

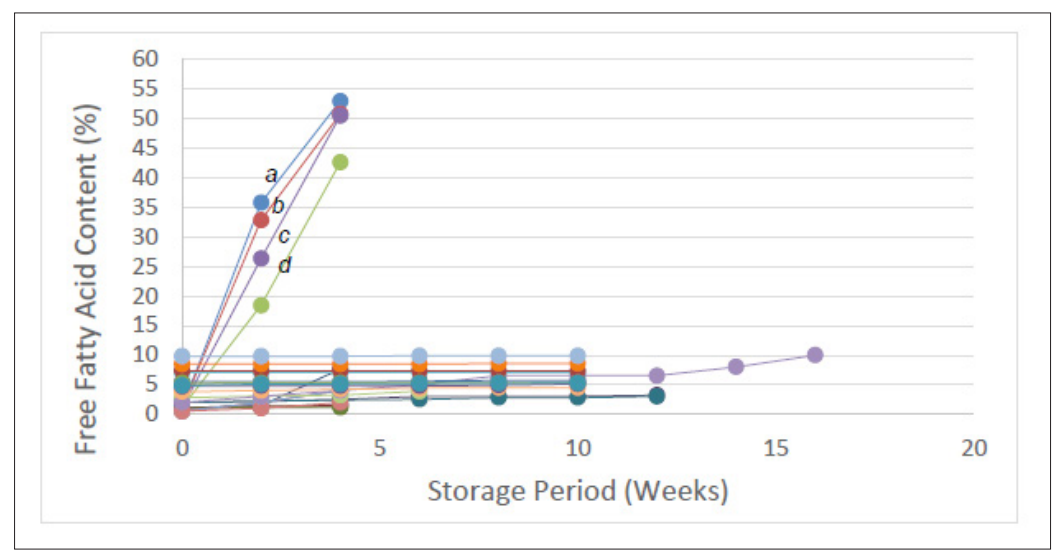

Figure 1: Free Fatty Acid Content over Storage Period for Microwave Stabilization. The data has been generated from the literature (Patil et al., 2016; Kim et al., 2014; Bagchi et al., 2014; Lakkakula et al., 2004; Ramezanzadeh 1998; Begum et al., 2015). Individual color represents a single data set.

Infrared heating: Figure 2 below shows the data for infrared stabilized rice bran and its free fatty acid content over a storage period of up to 10 weeks. The FFA content of all samples in week- 0 is between 4.4 and $4.86 \%$, however, after 4 weeks of storage, all samples measured above the acceptable 5\% FFA content, deeming them unfit for human consumption. Although from these results it appears infrared is not an effective and suitable method for rice bran stabilization, the data shows that the technique has some potential for further investigations into its suitability. Only $33.3 \%$ of samples doubled in their FFA content within the first 4 weeks of storage. Comparing this to microwave heating where $36 \%$ of samples tested doubled in FFA content within the first 4 weeks of storage. Yilmaz et al. (2013) states that the most suitable treatment is an IR power of $700 \mathrm{~W}$ for 7 minutes, where as Irakli et al. (2018) states that infrared heating at $140^{\circ} \mathrm{C}$ for 15 minutes is the most effective stabilization method. To effective use of the data for scale up a process, an energy consumption approach is supposed to be more effective.

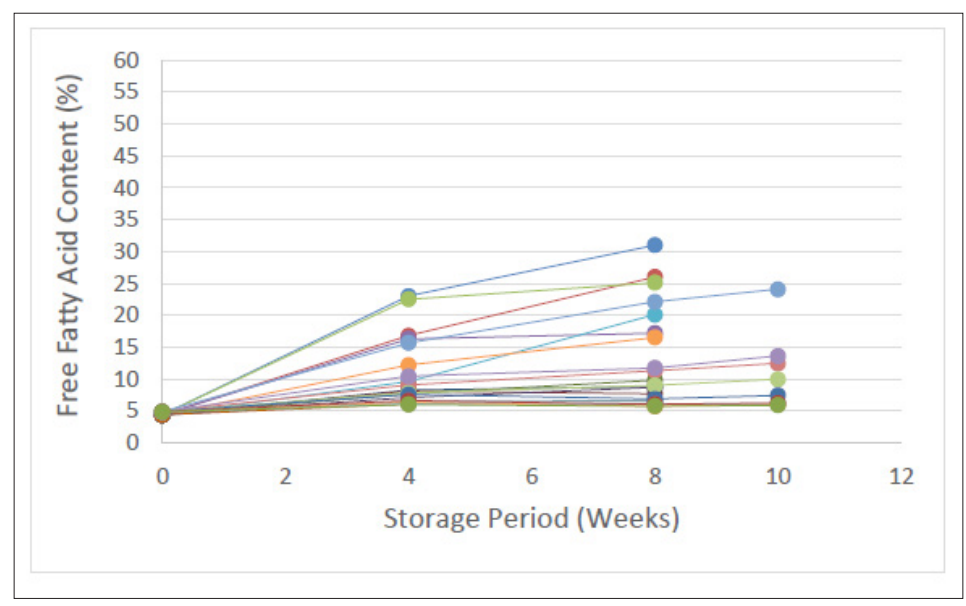

Figure 2: Free Fatty Acid Content over Storage Period for Infrared Stabilization. The data has been generated from the literature (Irakli et al., 2018; Y1lmaz et al., 2013; Y1lmaz 2016). Individual color represents a single data set. 
Energy consumption: As aforementioned, the energy consumption of a system is an incredibly effective method for comparing the performance of multiple techniques, relative to each other. Therefore, where possible calculations have been carried out to find the energy consumption for each system, per kilogram $(\mathrm{kJ} / \mathrm{kg})$. The energy consumption per unit mass is important as almost all research in literature has been carried out using different sample sizes. Applying the same treatment in terms of energy to a smaller sample will tend to yield better results in terms of stability. Therefore, it is important to consider the energy per unit mass. As most data in the literature does not contain energy consumption data, the following calculation was made. To find energy consumption per unit mass for microwave treatment:

$\frac{\text { Energy Consumption }(K J)}{\text { Unit Mass }(K g)}=\frac{\text { Power }(W) \times \text { Treatment time }(s)}{\text { Mass of Sample }(K g)}$ Eq (1)
Figure 3 below shows a plot of the free fatty acid content of the stabilized microwave samples, at 0 weeks and at 4 weeks, against the energy consumption per kilogram $(\mathrm{kJ} / \mathrm{kg})$. Trendlines have been added to the figure in order to assess the data. It was expected that as more energy per unit mass was applied to the sample, the increase in free fatty acid content would reduce. Generally, this trend was followed with the data, however, all data did not correlate perfectly as expected. For data points below $720 \mathrm{~kJ} / \mathrm{kg}$, the free fatty acid content of some samples was over 20 times larger than other samples with the same energy per kilogram applied. Therefore, no confident conclusions can be drawn from the data below this energy threshold. However, from the trendlines plotted, it can be noted that a microwave treatment with an energy consumption of around $2000 \mathrm{~kJ} / \mathrm{kg}$ will stabilize the bran where the free fatty acid content increase over a period of 4 weeks will be almost negligible.

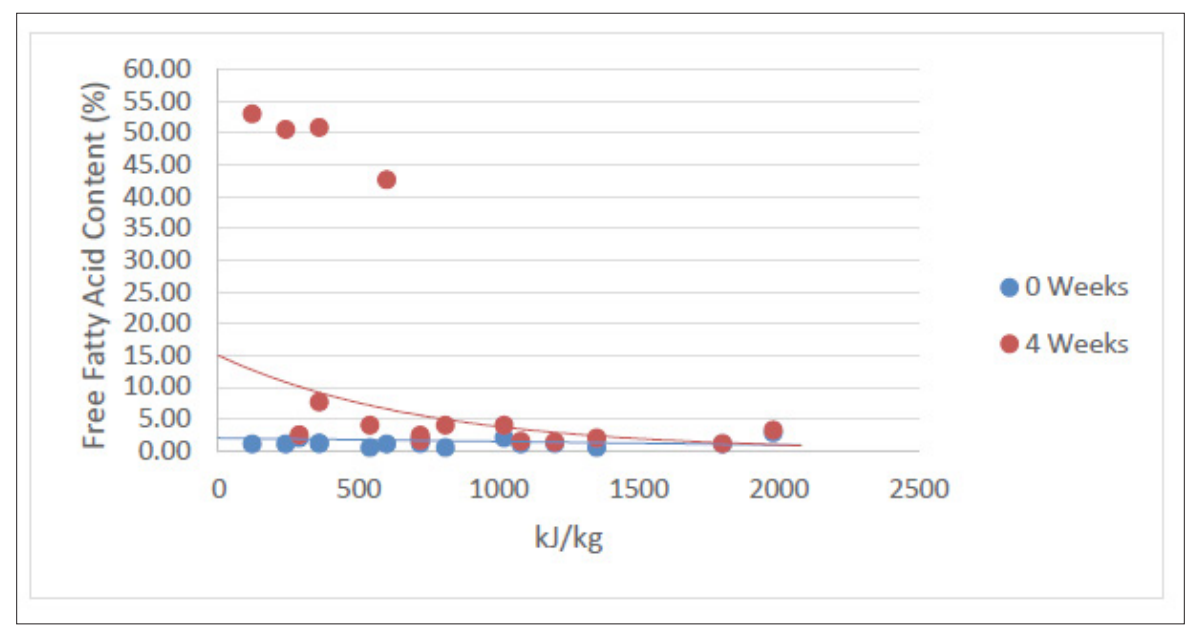

Figure 3: Relationship between energy consumption and the stability of rice bran for microwave stabilization. The data has been calculated from the available literature (Patil et al., 2016; Kim et al., 2014; Bagchi et al., 2014; Lakkakula et al., 2004; Ramezanzadeh 1998; Begum et al., 2015).

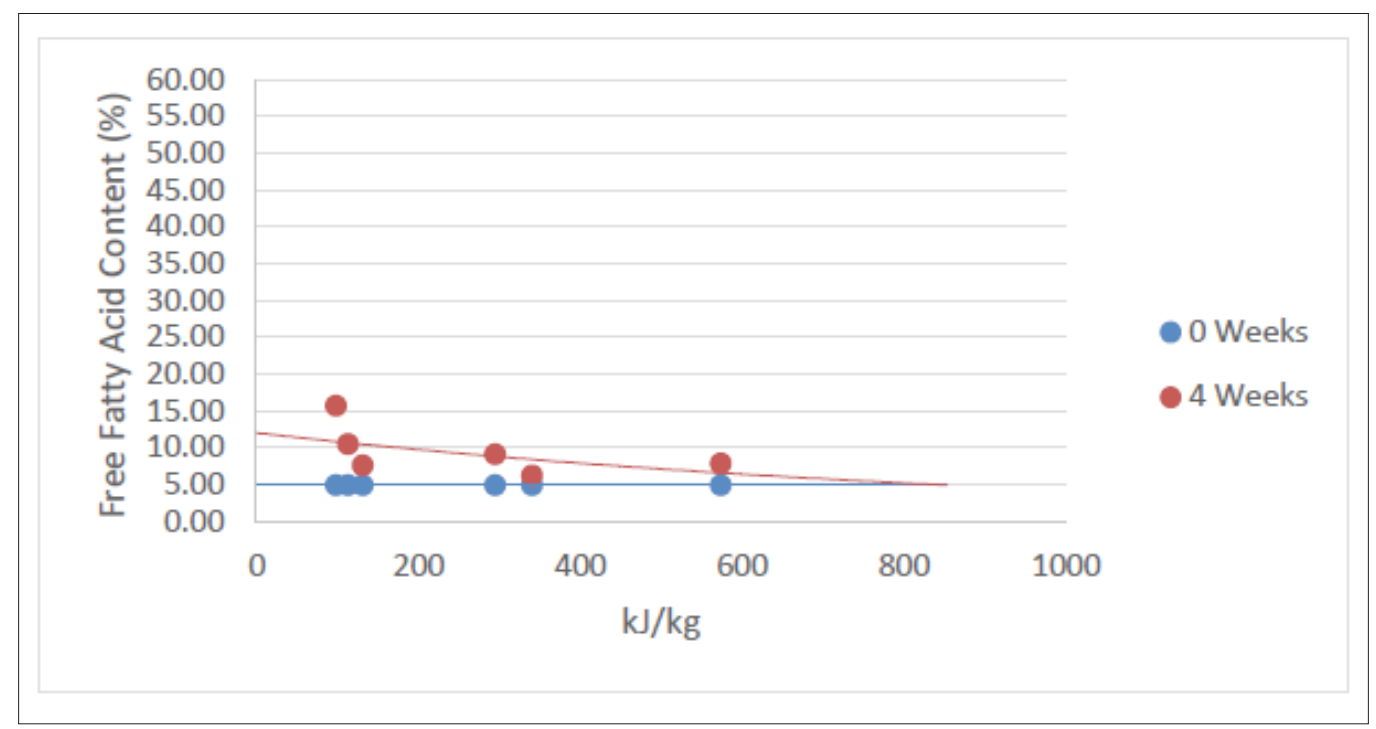

Figure 4: Relationship between energy consumption and the stability of rice bran for infrared stabilization. The data has been generated from the literature (Irakli et al., 2018; Y1lmaz et al., 2013; Y1lmaz 2016). 
For infrared energy, the information available regarding the energy consumption of the treatments was again mostly unavailable. The systems used to carry out the infrared treatments were moving conveyor systems, unfortunately, the information given in the reports was not sufficient to be able to accurately calculate the energy consumption and the mass of each sample. Figure 4 below shows a plot of the free fatty acid content of the stabilized infrared samples (where possible), at 0 weeks and at 4 weeks, against the energy consumption per kilogram $(\mathrm{kJ} / \mathrm{kg})$. Again, as the trendline shows, the data generally follows the trend that as more energy is applied to the sample in the stabilization treatment, however, the correlation is not perfect. The trendlines suggest that an infrared treatment with energy consumption at around $850 \mathrm{~kJ} / \mathrm{kg}$ will stabilize the bran where the free fatty acid content increase over a period of 4 weeks will be almost negligible, however, more data would be required to back this statement up. To conclude, for infrared heating, the lack of data available regarding the energy consumption limits the conclusions that can be drawn from the study. Therefore, more work will need to be carried out in order to fully assess the effects of infrared treatment by energy consumption.

From all the data gathered and presented in this section of the report, some conclusions can be drawn upon the most suitable method for rice bran stabilization. However, as aforementioned, the lack of emphasis placed upon the energy consumption of each stabilization method in the literature supports the idea in which more work needs to be carried out to confirm this conclusion.

From preliminary processing of the data found in literature, the stabilization methods deemed to be the most effective were microwave heating and dry heating. Where the initial FFA\% was below $5 \%$ for microwave heating, $87.5 \%$ of treatments were able to stabilize the bran to within the 5\% FFA\% threshold in the 4-week period. For dry heating, $50 \%$ of the treatments were able to stabilize the bran to within the 5\% FFA\% threshold.

The main aim of the study was to find the most suitable method for rice bran stabilization with respect to the energy required to achieve stability in the bran. From the information available in the literature and gathered through contact with the authors, it was visible that the energy consumption for each stabilization process was rarely considered. Therefore, an attempt was made to calculate, where possible, the energy consumption for each experiment presented in the literature. This was possible for two stabilization methods, microwave and infrared heating. From the figures plotted, using trendlines, for MW heating $2000 \mathrm{~kJ} / \mathrm{kg}$ was the energy consumption per unit mass which would be deemed to stabilize the bran completely within a 4-week period. For infrared heating $850 \mathrm{~kJ} / \mathrm{kg}$ was the energy consumption per unit mass which would be deemed to stabilize the bran completely within a 4-week period.

\section{Conclusion}

Presented here are the concise findings of the study into rice bran stabilization. The most effective method for stabilizing rice bran was concluded to be both microwave heating and dry heating. Both methods provided satisfactory free fatty acid content levels under specific conditions. For microwave heating, $87.5 \%$ of samples, where the FFA\% was under $5 \%$ were stabilized within the $5 \%$ threshold after a storage period of 4 weeks. Dry heating, where an anomaly result was removed, $60 \%$ of the samples were stabilized within the $5 \%$ threshold after a storage period of 4 weeks. However, in terms of energy consumption, infrared stabilization has been found to be effective methods.

\section{Acknowledgment}

The authors are thankful to EPSRC and Innovate UK for funding this project (EP/P026206/1) to be carried out.

\section{Conflict of Interest}

The authors hereby state that there is no conflict of interest to declare.

\section{References}

1. Ahmed F, Platel K, Vishwanatha S, Puttaraj S, Srinivasan K (2006) Improved shelf-life of rice bran by domestic heat processing and assessment of its dietary consumption in experimental rats. Journal of The Science of Food and Agriculture 87(1): 60-67.

2. Akhter M (2015) Inactivation of lipase enzyme by using chemicals to maximize rice bran shelf life and its edible oil recovery. Journal of Nutrition \& Food Sciences s12: 002.

3. Amarasinghe BMWPK, Kumarasiri MPM, Gangodavilage NC (2009) Effect of method of stabilization on aqueous extraction of rice bran oil. Food and Bioproducts Processing 87: 108-114.

4. Bagchi T, Adak T, Chattopadhyay K (2014) Process standardization for rice bran stabilization and its' nutritive value. Journal of Crop and Weed 10(2): 303-307.

5. Baum \& Whiteman (2018) Trend Reports of Baum \& Whiteman.

6. Begum A, Sarma J, Borah P, Moni BP, Saikia R, et al. (2015) Microwave (MW) energy in enzyme deactivation: Stabilization of rice bran from few widely consumed indigenous rice cultivars (Oryza sativa L.) from eastern himalayan range. Current Nutrition \& Food Science 11(3): 240245.

7. Borresen E, Ryan E (2014) Rice bran: A food ingredient with global public health opportunities. Wheat and Rice in Disease Prevention and Health, pp. 301-310.

8. Cicero A, Derosa G (2005) Rice bran and its main components: Potential role in the management of coronary risk factors. Current Topics in Nutraceutical Research 3(1): 29-46.

9. Gopinger E, Ziegler V, Catalan A, Krabbe E, Elias M, et al. (2015) Whole rice bran stabilization using a short chain organic acid mixture. Journal of Stored Products Research 61: 108-113.

10. Henderson A, Ollila C, Kumar A, Borresen E, Raina K, et al. (2012) Chemopreventive properties of dietary rice bran: Current status and future prospects. Advances in Nutrition 3(5): 643-653.

11. Irakli M, Kleisiaris F, Mygdalia A, Katsantonis D (2018) Stabilization of rice bran and its effect on bioactive compounds content, antioxidant activity and storage stability during infrared radiation heating. Journal of Cereal Science 80: 135-142.

12. Kahlon T, Chow F, Chiu M, Hudson C, Sayre R (1996) Cholesterol-lowering by rice bran and rice bran oil unsaponifiable matter in hamsters. Cereal Chem 73(1): 69-74.

13. Kim S, Chung H, \& Lim S (2014) Effect of various heat treatments on rancidity and some bioactive compounds of rice bran. Journal of Cereal Science 60(1): 243-248. 
14. Lakkakula N, Lima M, Walker T (2004) Rice bran stabilization and rice bran oil extraction using ohmic heating. Bioresource Technology 92(2): 157-161.

15. Loypimai P, Moonggarm A, Chottanom P (2009) Effects of ohmic heating on lipase activity, bioactive compounds and antioxidant activity of rice bran. Australian Journal of Basic and Applied Sciences 3(4): 3642-3652.

16. Lv S, He L, Sun L (2018) Effect of different stabilisation treatments on preparation and functional properties of rice bran proteins. Czech J Food Sci 36(1): 57-65.

17. Patil S, Kar A, Mohapatra D (2016) Stabilization of rice bran using microwave: Process optimization and storage studies. Food and Bioproducts Processing 99: 204-211.

18. Ramezanzadeh F (1998) Prevention of hydrolytic and oxidative rancidity and nutrient losses in rice bran during storage. Louisiana State University, USA.
19. Tao J (1989) Rice bran stabilization by improved internal and external heating methods. Louisiana State University, USA.

20. Tao J, Rao R, Liuzzo J (1993) Microwave heating for rice bran stabilization. Journal of Microwave Power and Electromagnetic Energy 28(3): 156164.

21. Yılmaz N, Tuncel N, Kocabıyık H (2013) Infrared stabilization of rice bran and its effects on $\gamma$ - oryzanol content, tocopherols and fatty acid composition. J Sci Food Agric 94(8): 1568-1576.

22. Yllmaz N (2016) Middle infrared stabilization of individual rice bran milling fractions. Food Chem 190: 179-185. 